\title{
DÜŞÜK HIZLI KABLOSUZ KİŞİSEL ALAN AĞLARINDA GÜRÜLTÜLÜ SİNYALLERE YÖNELİK ADAPTİF VERİ DİZİSİ ÜRETECİ
}

\author{
Sibel ARSLAN, Selçuk ÖKDEM \\ Erciyes Üniversitesi Mühendislik Fakültesi, Bilgisayar Mühendisliği Bölümü, Kayseri \\ sibel.arslan2@icisleri.gov.tr, $\underline{\text { okdem@ercives.edu.tr }}$
}

(Geliş/Received: 06.08.2014; Kabul/Accepted: 15.07.2015)

ÖZET

Kablosuz Kişisel Alan Ağları (PAN, Personal Area Networks), bilgisayarlar, telefonlar ve endüstriyel cihazlar arasında veri iletişiminde kullanılan bilgisayar ağlarıdır. $\mathrm{Bu}$ ağlar kısa iletişim mesafelerinde, düşük veri hızlarında, düşük güçte radyo sinyalleriyle çalışırlar. IEEE 802.15 .4 protokolü bu ağlarda yaygın olarak kullanılan bir standarttır. IEEE 802.15.4 protokolünün fiziksel katmanındaki her paket dört bitlik veri sembollerine ayrıştırılır ve her sembol otuz iki bitlik sözde rasgele gürültü dizisine (PN Chip Sequence) çevrilir. Bu makalede IEEE 802.15.4 protokolünde farklı kablosuz kanal türleri için birim zamanda maksimum veri iletimini gerçekleştirebilecek en iyi veri dizilerin bulunması amaçlanmıştır. Bunu gerçekleştirmek için adaptif bir veri dizisi üretme mekanizması önerilmiştir. IEEE 802.15.4 protokolünün mevcut ve önerilen PN dizilişleri ile MAC performans analizleri yapılmıştır. Önerdiğimiz metot, çip dizilerinin bit uzunluğunun adaptif yapıda ayarlayarak paket gönderim başarısını artırmıștır. Ortamdaki gürültü seviyeleri gözetilerek adaptif PN dizileri üretilmiștir. Bu diziler, standart IEEE 802.15.4 dizilerine kıyasla veri aktarım oranında dikkate değer artışlar sağlamıştır. Önerinin başarısı, karşılaştırmalı performans sonuçlarını gösteren tablolar ve grafiklerle açıklanmıştır.

Anahtar Kelimeler: Kablosuz kişisel alan ağları, ortam erişim kontrol performansı, gürültü benzeri çip dizileri, katmanlar arası haberleşme

\section{ADAPTIVE DATA SEQUENCE GENERATOR FOR NOISY SIGNALS IN LOW RATE WIRELESS PERSONAL AREA NETWORKS}

\begin{abstract}
Wireless Personal Area Networks (WPANs) are computer networks used for data transmission among devices such as computers, telephones and industrial devices. These networks are operated in short ranges using low data rates and low-power radio signals. IEEE 802.15.4 protocol is a common standard used in these networks. Each packet is spitted into symbols including four data bits, and each of these symbols translated to thirty two bits Pseudo Noise(PN) chip sequences in physical layer of IEEE 802.15.4 protocol. In this paper, it is aimed to find the best data chip sequences to obtain maximum data transfer in a time unit for different wireless channel types for IEEE 802.15.4 protocol. To accomplish this, an adaptive data sequence generator mechanism is proposed. Performance analyses of IEEE 802.15.4 protocol with traditional and proposed PN sequences are made. The proposed method increased the packet transmission success by adjusting the lenght of chip sequences. These sequences considerably increase data throughput compared to standart IEEE 802.15.4 sequences. The success of the proposal is illustarated in tables and graphs showing comparative performance results.
\end{abstract}

Keywords: Wireless personal area networks, media access control performance, pseudo noise chip sequences, cross-layer communication

\section{GİRIŞ (INTRODUCTION)}

Düşük Enerjili Düşük Hızlı Kablosuz Kişisel Alan Ağları (DH-KKA), küçük boyutlarda daha az enerji ile çalışan ağ yapılarıdır. Bu ağlar bina otomasyonu, sağlık durumu takibi, taşıt izleme sistemi, askeri gözlem ve deprem izleme uygulama alanlarında yaygın olarak kullanılmaktadır [1]. IEEE 802.15.4, DH-KKA' da yaygin olarak kullanılan bir protokoldür. Protokolde iki farklı çalışma modu 
desteklenir. Bunlar slotlu ve slotsuz haberleşme tipindedir. Slotlu haberleşmede koordinatör, bağlı olduğu elemanlara belirli süreler tanıyarak veri iletimini senkronize eder. Slotsuz haberleşmede ise elemanlar CSMA/CA (Carrier Sense Multiple Access/Collision Avoidance) yöntemi ile kablosuz ortamı kullanırlar. CSMA/CA, iletim halindeki verilerin çarpışmasının engellenmesi için kullanılır.

IEEE 802.15.4 fiziksel (PHY) katmanı $2450 \mathrm{MHz}$ frekans bandında $250 \mathrm{~Kb} / \mathrm{s}$ hızında O-QPSK (Dördün Faz Kaydırmalı Anahtarlama) tekniğini kullanarak veri aktarımı yapar. PHY katman protokol veri birimi (PPDU, Physical Protocol Data Unit)' nin tüm baytları veri sembolleri ile eşlenir. Her bir veri sembolü IEEE 802.15.4 standardında tanımlanan 32 bit sözde rasgele gürültü dizisi (PN Chip Sequences) ile eşlenerek çip dizileri elde edilir.

Kablosuz iletimde haberleşme mesafesi arttıkça alıcıya ulaşacak sinyal zayıflar. Zayıflayan sinyale iletim ortamındaki gürültü eklenir. Pasif IEEE 802.15.4 ağlarda, iletim kalitesini belirleyen Sinyal Gürültü Oranı (SNR, Signal Noise to Ratio) eklenen gürültünün sinyale etkisini ölçmek için kullanılır. Gürültü sinyalleri kanal boyunca iletilen çerçevelerde bit hatalarına neden olabilir.

$\mathrm{Bu}$ makalede, gürültü ortamları dikkate alınarak adaptif yapıda yeni gürültü dizileri araştırılmıştır. $\mathrm{Bu}$ diziler ile veri aktarım oranlarında artışlar gözlenmiştir. Sonuçlar, IEEE 802.15.4 protokolünde kullanılan standart gürültü dizileriyle karşılaştırılmıştır. Performans karşılaştırmalarından önerilen adaptif dizi üretme metodunun oldukça başarılı olduğu görülmüştür. Makale içeriğinde devam eden ikinci bölümde mevcut çalışmalardan bahsedilmiştir. Üçüncü bölümde IEEE 802.15.4 protokolünün işleyişi özetlenmiştir. Dördüncü bölümde sinyal güç oranının çalışmamızdaki yeri açıklanmıştır. Beşinci bölümde performans verilerinin alınması için geliştirilen uygulama yazılımından bahsedilmiştir. Devam eden bölümlerde ise performans sonuçlarına ve tartışmalara yer verilmiştir.

\section{MEVCUT ÇALIŞMALAR (RELATED WORKS)}

Gürültülü ortamlarda verimli haberleşmenin sağlanmasına yönelik birçok çalışma yapılmıştır. Bu çalışmaların çoğu IEEE 802.11 protokolü üzerinde yoğunlaşmıştır. $\mathrm{Bu}$ anlamda, S. Ci ve H. Sharif yapmış olduğu çalışmalarında IEEE 802.11 kablosuz ağ performansını analiz etmiş ve maksimum veri iletimini sağlamak için optimum çerçeve boyutu tahmin edilmeye çalışmışlardır [2]. Gürültülü ortamlarda veri paketlerini parçalayarak daha küçük boyutlarda göndermek pratik bir yöntemdir. Y.D. Lin ve arkadaşları WLAN performansını artırma yönünde bu yöntemi kullanmışlardır [3]. Bu yöntemi kullanan örnek diğer çalışmalar [4, 5] de IEEE 802.11 ağlar için uygulanmıştır. Ancak, bu çalışmalar IEEE 802.11 protokolüne yönelik olup IEEE 802.15.4 haberleşme sistemlerine uygun değildir. B.S. Kim, Y. Fang ve T.F. Wong yapmış olduğu çalışmalarında IEEE 802.11 standardında kanal bilgisi ve değişken boyutta çerçeve iletimini temel alarak dinamik çerçevelemeye uyumlu protokol geliştirilmeyi amaçlamıştır [6]. Optimum çerçeve boyutunu bulmak için bir diğer alternatif yöntem, her başarısız iletim sonrası çerçeve boyutunun yarıya indirilmesi ve her başarılı iletim sonrası çerçeve boyutunun ikiye katlanmasıdır. S. Ci ve H. Sharif çerçeve boyutunu ikiye bölme/katlama yoluyla en uygun boyutu bulmaya çalışmışlardır [7]. Bu anlamda $[8,9]$ nolu kaynaklarda verilen çalışmalar IEEE 802.11 protokolü için düzenlenmiş olup IEEE 802.15.4 protokolüne uygun çalışmalarda bulunulmamıștır. Kanal özelliklerinin istatistiksel değerlerini kullanarak verimli haberleşmeyi hedefleyen çalışmalarda yapılmıştır $[10,11]$. Ancak, bu çalışmalar IEEE 802.15.4 protokolünün fiziksel ve ortam erişim katmanlarına uyumsuzluk göstermektedir.

IEEE 802.15.4 PHY ve MAC alt katman tanımlamaları IEEE 802.11 standardındaki tanımlamalardan farklı olduğundan dolayı bu çalışmalar IEEE 802.15.4'e uyumlu değildir. T. Sato, M. Kawabe, T. Kato ve A. Fukasawa yapmış olduğu çalışmalarında, üç-durumlu Markov modeli kullanılarak kanal patlama hatası altında hibrit otomatik tekrar isteği (ARQ) için bir verim analizi yöntemi tanımlamışlardır. $\mathrm{Bu}$ çalışmada slotlu CSMA/CA kullanılmıştır [12]. Ancak, slotsuz CSMA/CA kullanımı hakkında bilgi verilmemiştir. H.S. Wang ve P.C. Chang yapmış olduğu çalışmalarında, slotsuz IEEE 802.15.4 MAC (Media Access Control, Ortam Erişim Kontrolü) fonksiyonları için olasılık tabanlı bir model geliştirmiştir. Bu modelde, çarpışmalar için detaylı bir analiz yapılmasına rağmen, gürültü sinyalleri etkili kanallar için bir çalışma sunulmamıştır [13]. F.Shu ve T.Sakaurai yapmış olduğu çalışmalarında IEEE 802.15.4 MAC protokolü enerji korumalı CSMA/CA kullanarak kablosuz algılayıcı ağların performansı hakkında analitik sonuçlar elde etmişlerdir. Geliştirilen modelde doymuş ve doymamış ağlar için sonuçlar alınmasına rağmen gürültülü ortamlara ilişkin performans analizi yapılmamıştır [14]. M. Goyal ve arkadaşları tarafindan yapılan çalışmada slotsuz IEEE 802.15.4 MAC protokolü için olasılık tabanlı bir model geliştirilmiştir. $\mathrm{Bu}$ çalışmada veri iletim gecikmesi ve veri paketi kayıp oranı modellenmesine rağmen IEEE 802.15.4 protokolünün DSSS modülasyon tekniğine ilişkin verileri çalışmada kapsam dışı bırakılmıştır [15]. J. Misic, S. Shafi ve V. Misic yapmış oldukları çalışmada, IEEE 802.15.4 slotlu modda çalışan kişisel alan ağını, ayrık zaman Markov zincirleri kullanarak analiz etmişlerdir [16]. Ancak bu çalışmada, ortam gürültüsünün performansa etkisi incelenmemiștir. J. Zhu, Z. Tao ve C. Lv yapmış oldukları çalışmada heterojen doymamış trafik için CSMA/CA algoritmasının performansı 
değerlendirmişlerdir. $\mathrm{Bu}$ çalışmada slotlu IEEE 802.15.4 haberleşmesinde doymamış durum analizinde performans incelemesi yapılmasına rağmen slotsuz IEEE 802.15.4 haberleşmesine ilişkin değerlendirme yapılmamıştır [17]. Markov modelleri kullanılarak paket gönderim hatalarını dikkate alan yöntemler [18,19] önerilmiş ama bunların IEEE 802.15.4' e uyarlamaları yapılmamıștır. C. Y. Lee, H. Cho, G. U. Hwang, Y. Doh ve N. Park '1n yapmış oldukları çalışmada IEEE 802.15.4 onaylı ve slotlu CSMA/CA kablosuz algılayıcı ağları için analitik model geliştirilmiştir [20]. Ancak, çalışma IEEE 802.15.4 protokolünün slotsuz modu hakkında herhangi bir bilgi içermemektedir. P. Park ve arkadaşları yapmış oldukları çalışmada [21] ve P.D. Marco, P. Park, C. Fischione, K. H. Johansson yapmış oldukları çalışmada [22] slotsuz IEEE 802.15.4 MAC operasyonlarını stokastik yapıda modellemişlerdir. $\mathrm{Bu}$ çalışmalarda, görev döngüsü (duty-cycle), çarpışma ve ayrıntılı trafik hızı gibi parametrelerin etkisini analiz edilmiş, ancak gürültü sinyalleri tarafindan etkilenen kanallar için bir araştırma dâhil edilmemiştir. F. Wang, D. Li, Y. Zhao, düğüm ve kanal durumlarını bulunduran ayrik zaman Markov zincirinin kullanıldığı haberleşmelerde performans analizini yapmışlardır [23]. Ancak, gürültü sinyallerinin protokolünün performansı üzerindeki etkisiyle ilgili bir araştırmada bulunmamışlardır.

$\mathrm{Bu}$ makalede, rastgelelik özelliği sağlayan yeni gürültü dizileri (pseudo-noise sequence) önerilmiştir. Önerilen bu diziler ile gürültülü ortamlarda daha verimli bir haberleşme sağlanmıştır. Mevcut IEEE 802.15.4 protokolünün sabit uzunlukta tanımlanmış dizilerine, komşu bitlerin üzerine eklenmesi/çıkarılması yoluyla bu öneri gerçekleştirilmiştir (Şekil 1). Önerdiğimiz yeni diziler, farklı gürültü seviyelerindeki paket gönderim başarısını arttırmıştır. Önerilen yaklaşım, adaptif yapıda gürültü seviyelerini dikkate alarak optimum diziler üretmiştir. Bu sayede, veri aktarım oranında iyileşmeler sağlamıştır. $\mathrm{Bu}$ yaklaşımın başarısı mevcut IEEE 802.15.4 protokolü ile karşılaştırılmış ve alınan performans sonuçları tablo ve şekillerle açıklanmıştır.

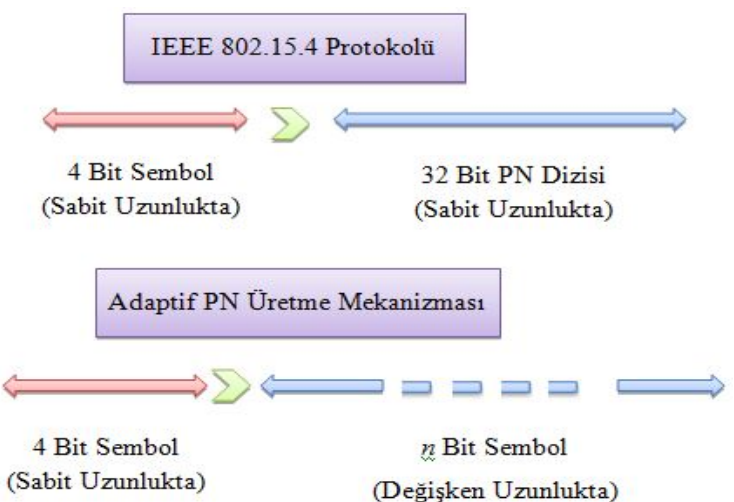

Şekil 1. Adaptif PN dizisi üretme (Adaptive PN sequence generation)

\section{IEEE 802.15.4 PROTOKOLÜ (IEEE 802.15.4 PROTOCOL)}

IEEE 802.15.4 LR-WPAN k1sa mesafede veri aktarımını sağlamayı amaçlayan güvenilir yapıda bir iletişim ağıdır. LR-WPAN yıldız topolojisi veya noktadan noktaya ağ topolojisinin birinde işletilebilir. Noktadan noktaya ăg topolojisinde, yıldız topolojisindeki iletişimden farklı olarak, ağ içindeki cihazlar menzili içindeki diğer cihazlar ile haberleşebilir.

IEEE 802.15.4 standardının PHY katmanı, güncel kanal için enerji sezimi (ED), alınan paketler için bağlantı kalite bildirimi (LQI), CSMA-CA için temiz kanal atanması (CCA), kanal frekans seçimi görevlerden sorumludur.

IEEE 802.15.4 PHY 2450 Mhz bandında 16 kanal, $915 \mathrm{Mhz}$ bandında 10 kanal ve $868 \mathrm{Mhz}$ bandında 1 kanala sahiptir. Uygulamalarda en çok $2.4 \mathrm{GHz}$ tercih edilir. Bu frekansta veri aktarım hızı $250 \mathrm{~Kb} / \mathrm{s}$, kod iletim hızı 2M çip/s olarak tanımlanır.

$2450 \mathrm{MHz}$ PHY katman, O-QPSK modülasyonunu kullanır. PHY katman protokol veri biriminin (PPDU) tüm baytları modülasyon ve yayılma fonksiyonları ile aynı işleme tabi tutulmaktadır. Her bir baytın en düşük anlamlı bitleri (4 LSB' si $\left(b_{0}, b_{1}, b_{2}, b_{3}\right)$ ) bir veri sembolü ile ve sonraki en anlamlı bitleri (4 MSB' si $\left.\left(b_{4}, b_{5}, b_{6}, b_{7}\right)\right)$ bir sonraki veri sembolü ile eşlenir. İşlem, sıralı olarak senkronizasyon başlığı ve başlangıç alanı ile başlar ve PSDU (Physical Layer Service Data Unit)' nun son bayt1 ile biter.

Her bir veri sembolü, Tablo 1' de gösterilen IEEE 802.15.4 standardında tanımlanmış 32 - çip sözderasgele gürültü dizisi (PN sequences) ile eşlenerek çip dizileri türetilir. Sözde-rasgele gürültü dizileri içindeki en düşük anlamlı çip $\left(\mathrm{c}_{0}\right)$ en başta gönderilir. Çip dizileri yarım dalga sinüs darbe şeklini içeren O-QPSK modülasyonuyla taşıyıcı üstüne modüle edilir.

IEEE 802.15.4 standardının MAC alt katmanı fiziksel radyo kanalına erişimi, slot çerçevelerinin senkronize edilmesi, PAN' a bağlanma ve ayrılmanın desteklenmesi, cihaz güvenliği desteklenmesi, kanal erişimi için CSMA-CA mekanizmasını işletmesi, GTS mekanizmasının işletilmesi ve korunması gibi görevlerden sorumludur.

IEEE 802.15.4 protokolü MAC alt katmanını iki çalışma modu vardır. Bunlar slotlu (beacon enabled) ve slotsuz (beaconless) iletim modlarıdır. Slotsuz iletim modunda ağda bulunan cihazlar senkronizasyon için slotsuz CSMA-CA mekanizması kullanarak iletim yaparlar [18]. Şekil 2' de slotsuz iletim modunda CSMA-CA algoritma şeması verilmiştir. Algoritma, aUnitBackoffPeriod sembol süresine eşit olması gereken gecikme peryotları ismi ile anılan zaman birimleri kullanılarak işletilir. 
Tablo 1. Sembol - Çip eşlemesi (Symbol-Chip matching) [24]

\begin{tabular}{|c|c|c|}
\hline $\begin{array}{c}\text { Sembol } \\
\text { (Onlu) }\end{array}$ & $\begin{array}{l}\text { Sembol (Íkili) } \\
\left(\mathbf{b}_{0}, \mathbf{b}_{1}, \mathbf{b}_{2}, \mathbf{b}_{3}\right)\end{array}$ & $\begin{array}{c}\text { Çip Değerleri } \\
\left(\mathbf{c}_{0}, \mathbf{c}_{1}, \ldots, \mathrm{c}_{30}, \mathrm{c}_{31}\right)\end{array}$ \\
\hline 0 & 0000 & 11011001110000110101001000101110 \\
\hline 1 & 1000 & 11101101100111000011010100100010 \\
\hline 2 & 0100 & 00101110110110011100001101010010 \\
\hline 3 & 1100 & 00100001011100110110011100000110101 \\
\hline 4 & 0010 & 0101000100010011101101100111000011 \\
\hline 5 & 1010 & 00110101001000101110110110011100 \\
\hline 6 & 0110 & 11000011010100100010111011011001 \\
\hline 7 & 1110 & 10011100001101010010001011101101 \\
\hline 8 & 0001 & 10001100100101100000011101111011 \\
\hline 9 & 1001 & 10111000110010010110000001110111 \\
\hline 10 & 0101 & 01111011100011001001011000000111 \\
\hline 11 & 1101 & 01110111101110001100100101100000 \\
\hline 12 & 0011 & 00000111011110111000110010010110 \\
\hline 13 & 1011 & 0110000000111101111011100011001001 \\
\hline 14 & 01111 & 10010110000001110111101110001100 \\
\hline 15 & 1111 & 11001001011000000111011110111000 \\
\hline
\end{tabular}

Slotsuz CSMA-CA' da tüm cihazlar her bir iletim işlemi için $\mathrm{NB}$ ve $\mathrm{BE}$ ile isimlendirilen 2 değişken bulundurur. NB, güncel iletim denenirken CSMA-CA algoritmasının gerek duyduğu gecikme periyotları sayısıdır. Bu değer her yeni iletim işleminden önce 0 değeri ile başlatılır. BE, gecikme periyodu üstel değeri, cihazın kanal değerlendirmesi yapmadan önce ne kadar gecikme periyodu bekleyeceği ile ilişkilidir. macMinBE değeri 0 olarak ayarlanmış ise bu algoritmanın birinci iterasyonu müddetince çarpışmadan kaçınma geçersiz olacaktır.

$\mathrm{Bu}$ algoritmanın kanal değerlendirme kısmı süresince cihazın alıcısı aktif olduğu halde, cihaz bu zaman diliminde alınan tüm çerçeveleri imha edecektir. MAC alt katmanı NB ve BE değişkenlerini başlatır ve doğruca 2. adımı işletir. MAC alt katmanı 0 ile $2^{\mathrm{BE}}-1$ aralığında rasgele sayıda gecikme periyodu bekler ve sonra PHY katmanından CCA uygulaması ister. Eğer kanal meşgul ise MAC alt katmanı $\mathrm{NB}$ ve $\mathrm{BE}$ değişkenlerini bir arttıracaktır, burada $\mathrm{BE}$ ' nin aMaxBE değerinden büyük olmadığından emin olunmalıdır. Eğer NB değişkeni değeri macMaxCSMABackoffs değerine eşit veya daha küçükse, CSMA-CA algoritmada 2. Adıma geri dönecektir. NB daha büyükse, CSMA-CA algoritması kanal erişimi başarısız durumu ile sonlandırılacaktır. Eğer kanal boş olarak değerlendirilirse MAC alt katmanı çerçevenin iletimine hemen başlayacaktır.

\section{SINYAL GÜÇ ORANI (SIGNAL TO NOISE RATIO)}

IEEE 802.15.4 yönlendirme protokolünde düğümler arası yönlendirme maliyeti atamaları gerekir. Uzaklık birimi, bağlantı güvenilirliği, bağlantı gecikme süresi, bağlantının iletme/alma işlemindeki enerji tüketimi yönlendirme maliyeti ölçütlerindendir. Bağlantı güvenilirliği paketin başarılı/hatalı gönderim oranına veya beklenen iletim sayısıyla (ETX-bağlantı sonunda başarılı gönderilen paket sayısı) ölçülebilir [25].
Genellikle, bağlantı güvenilirliği Alınan Sinyal Güç Göstergesi (RSSI) ile ifade edilir. Alınan Sinyal Güç Göstergesi (RSSI) iletişim kanalındaki paketin iletimi boyunca radyo enerji göstergesidir. Radyo enerji sinyal enerjisi içerdiği kadar gürültü enerjisini de içerir [26].

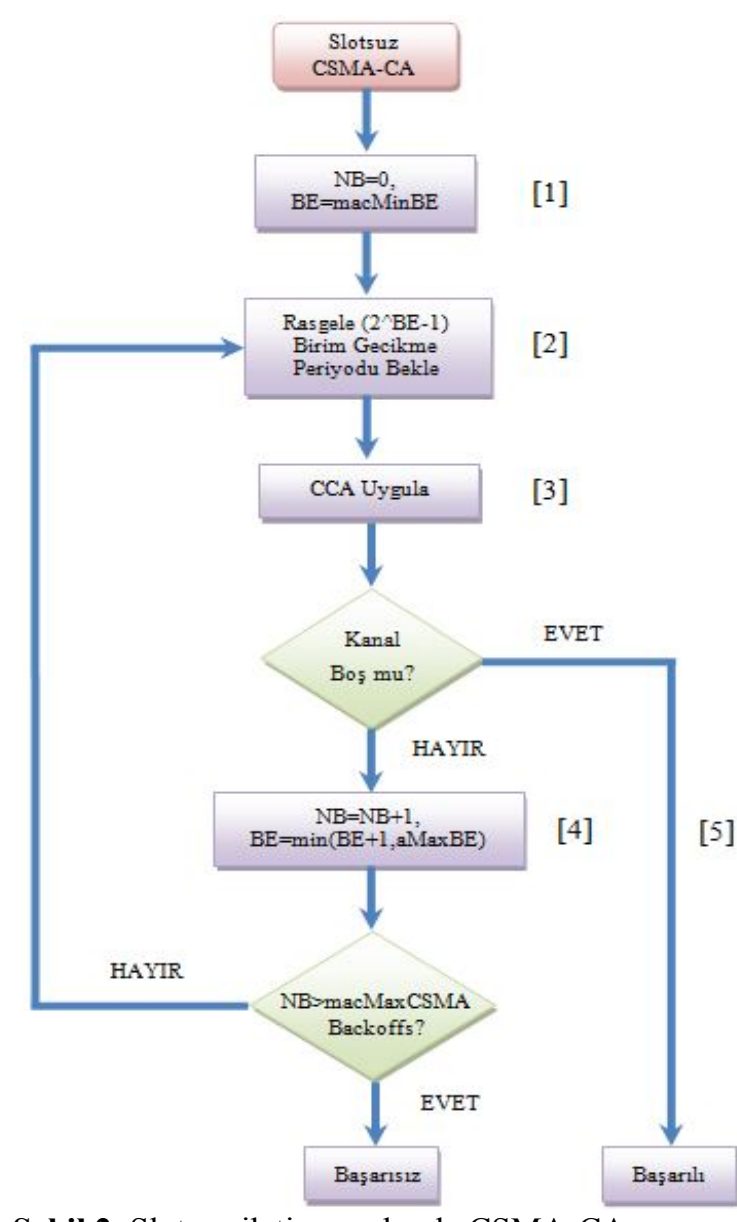

Şekil 2. Slotsuz iletim modunda CSMA-CA algoritması (CSMA/CA algorithm with unslotted mode) 
RSSI sinyal enerjisini belirlemede Sinyal Güç Oranı (SNR) eklenmesi gerekir. Sinyal Güç Oranı (SNR) iletişim kanalındaki enerji düzeylerinde sinyal ve güç oranı olarak tanımlanır. Sinyal gücünün azalıp gürültü gücünün artması durumunda iletişim kalitesi azalır ve sinyallerde bozulmalar artar.

Modülasyon tekniklerine göre SNR hesaplamasını belirleyen fonksiyonlar Tablo 2' de verilmiştir.

\section{PERFORMANS VERILERININ ALINMASI (OBT AINING PERFORMANCE DATA)}

$\mathrm{Bu}$ bölümde alınan sonuçlar, istatistiki açıdan anlamlı değerler üretmesi amaciyla minimum \%95 güvenirlilik aralığını sağlayacak şekilde çoklu defa koşturulmuştur. Ortalama sonuçlar alınırken, bu aralığın sağlanabilmesinde simülasyonun en az 30 defa koşturulduğu gözlenmiştir.

Tablo 2. SNR hesaplanırken kullanılan fonksiyonlar (Functions used for calculation of SNR) [27]

\begin{tabular}{|c|c|}
\hline Fonksiyon & Matematiksel İfade \\
\hline Q Fonksiyonu & $Q(x)=\frac{1}{\sqrt{2 \pi}} \int_{x}^{\infty} \exp \left(-\frac{t^{2}}{2}\right) d t$ \\
\hline Marcum Q & $Q_{M}(a, b)=\int_{b}^{\infty} t * \exp \left(-\frac{t^{2}+a^{2}}{2}\right) I_{M-1}(a t) d t$ \\
Fonksiyonu & $J_{v}(z)=\sum_{k=0}^{\infty} \frac{(z / 2)^{v+2 k} \cdot(-1)^{M}}{k ! \Gamma(v+k+1)}$ \\
\hline Birinci & dereceden \\
Modifiye & Burada Gama Fonksiyonu: \\
Edilmiş Bessel & $\Gamma(x)=\int_{0}^{\infty} e^{-t} t^{x-1} d t$ \\
Fonksiyonu & \\
\hline
\end{tabular}

Koşturulan yazılımda performansı belirleyen MAC parametre değerleri Monte Carlo Metodu kullanılarak elde edilmiştir. Bu çalışmada IEEE 802.15.4 karşılıklı olarak veri transferinde bulunan noktadan noktaya ağ topolojisi ve slotsuz CSMA-CA mekanizmasi kullanılmıştır. IEEE 802.15.4 2003 versiyonu kullanılarak maksimum çerçeve boyutlarında $2.4 \mathrm{GHz}$ frekans bandında iletişim gerçekleştirilmiştir. Her bir gürültü ortamı için algoritma 30 defa koşturulmuş ve bunlara ilişkin ortalama değerler alınmıştır. IEEE 802.15.4 standardı gönderilecek her 4 bilgi bitini bir veri sembolüyle eşleştirir. Eşleştirilen her sembol 32 çip bitiyle temsil edilir. Adaptif yayılım faktörü üretme mekanizmasında her bir sembol için 5, 6, 7, .. ,58 bitlerinden oluşan çip bitleri kullanılır. Farklı çip bitleri IEEE 802.15.4 standardında yer alan 32 çip bit tablosuna komşu bitlerin eklenip çıkarılmasıyla elde edilmiştir. Şekil 3, Şekil 4 ve Şekil 5'de yayılım faktörlerinin elde edilmesi gösterilmiştir. Adaptif PN dizisi üretme mekanizmasında;
1. Çip bit uzunluğu 32 den küçük ve eşit olan PN dizileri, standartta tanımlanan PN dizisinden türetilir. Türetme işlemi, komşuluk bilgisi rehberliğiyle gerçekleştirilir. Yayılım faktöründe belirlenen oranda yer alan değerler ölçüsünde $\mathrm{PN}$ dizisinin uzunluğu hesaplanir.

2. Çip uzunluğu 32 den büyük olan PN dizilerinde ise standartta tanımlanan PN dizisinden ne ölçüde fazlalık bulunuyorsa, mevcut PN dizisine o ölçüde ilave edilmesiyle yeni dizi oluşturulur. Yayılım faktöründe belirtilen oranda yer alan değerler ölçüsünde boyutta artırım işlemi gerçekleştirilir.

Yukarıda verilen her iki durumu açıklayan örnek oluşumlar Şekil 3 ve Şekil 4'de verilmiştir. Şekil 3'de görüldüğü üzere yayılım faktörünün büyüklüğü ölçüsünde dizi genişliği ayarlanmaktadır. Şekilde yayılım faktörünün bazı örnek değerleri için dizilerin nasıl oluştuğunu gösteren yöntem açıklanmıştır. Şekil 4'de benzer durumun dinamik yapida gerçekleştirildiği görülmektedir. Gürültü oranı arttıkça dizi genişlemekte ve o oranda yayılım faktörü de artmaktadir.

Gürültü seviyesi az ortamlar için daha az çip bitleriyle, gürültü seviyesi çok ortamlar için daha çok çip bitleriyle temsil edilen rasgele diziler, daha hizlı veri aktarımını sağlamaktadır. Sayısal değerlerin alınmasında Monte Carlo metoduyla işletilen adımlar aşağıda verilmiştir.

\section{Adım 1. Başlatma:}

- Popülasyon sayısını (n) 26’ ya (Chip Hata Oranı $\in$ $[0,0.25])$ set et, popülasyondaki her bir komşuluk için (neighbour) 54' e ve bireyi temsil eden indeksi (packet) 128' e set et,

Adım 2. Popülasyondaki her komşuluk için aşă̆ıdaki işlemleri yap:

Adım 2.1. Popülasyondaki her birey için aşă̆ıdaki işlemleri uygula:

- MAC çerçeveler için 4 bitlik rasgele semboller üret, bu sembollere ilişkin adaptif kod üretme mekanizması ile kodlamayı gerçekleştir. Sağlıklı giden veri miktarını ve iletim süresini hesapla,

Adım 3. Durdurma kriterini değerlendir.

Eğer durdurma kriteri sağlandı ise sonuç verilerini yazdır ve çık

- Durdurma kriteri: (bütün komşuluk değerleri için bu hesaplama yapıldı mı?).

- Her komşuluk değeri için veri aktarım oranının en iyi olduğu yayılım faktörünü not et. 


\section{Adım 4. Diğer durumda çalışmaya devam et,}

Popülasyon komşuluk değerini (neighbour) 1 artır ve adim 2' ye git.
$\mathrm{Bu}$ adımlar farklı gürültülü ortamları temsil eden farklı bit hata oranları için gerçekleştirilmiştir. Sonuçlar alındıktan sonra karşılaştırmalar ve grafiğe aktarma işlemi devam eden bölümde verilmiştir.

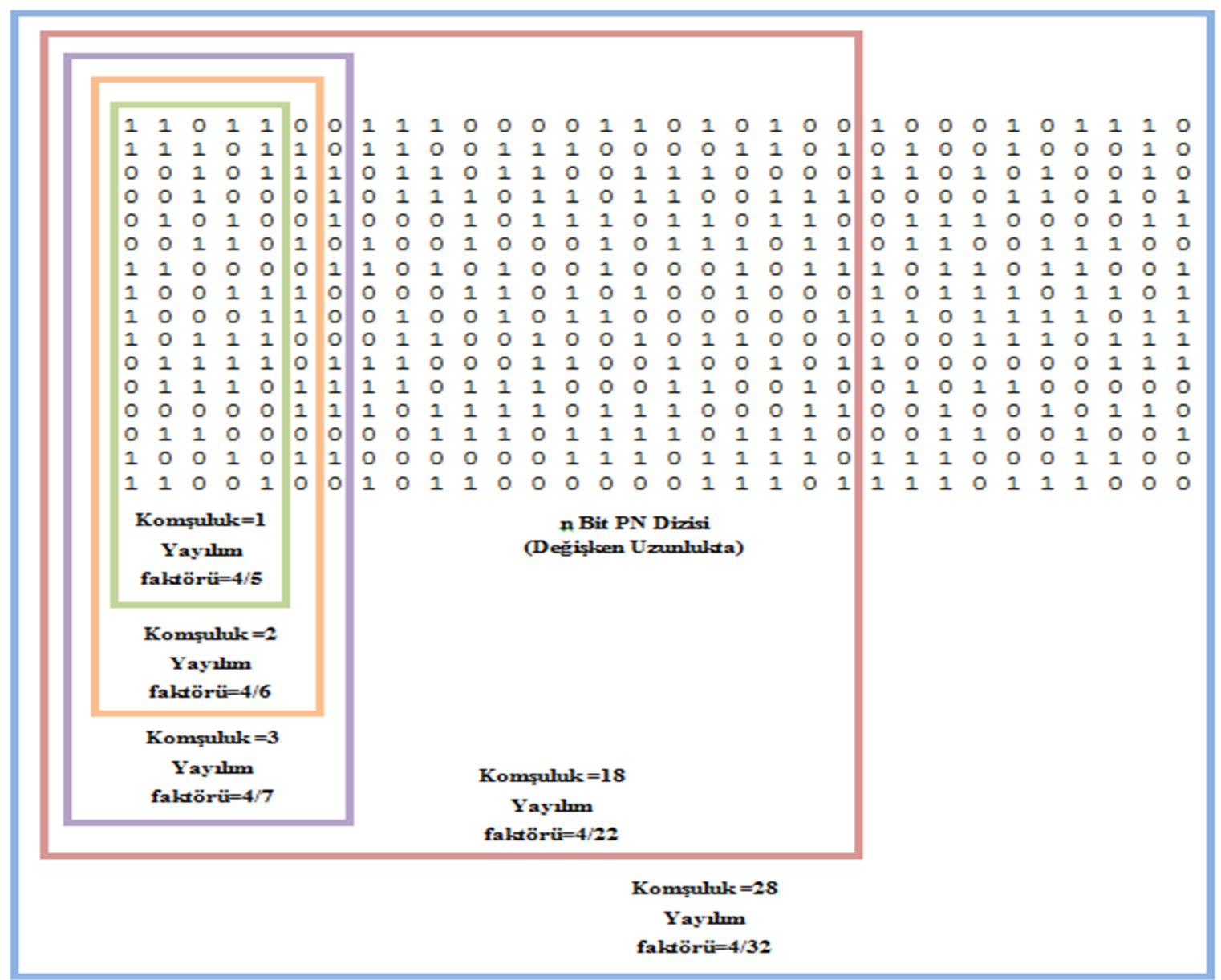

Şekil 3. İlk 28 komşuluk için adaptif PN dizisi üretme mekanizması (Adaptive PN sequence generator mechanism for the first 28 neighborhood)

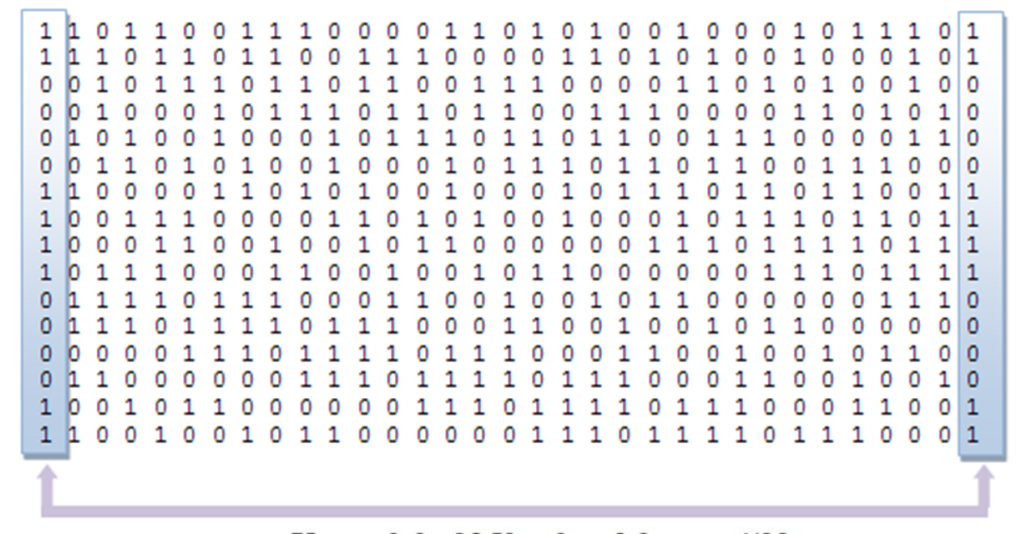

Komșuluk=29 Y aylım faktörü=4/33

Şekil 4. İlk 29 komşulukta adaptif PN dizisi üretme mekanizması (Adaptive PN sequence generator mechanism for the first 28 neighborhood) 


\section{UYGULAMA YAZILIMININ SONUÇLARI VE DEĞERLENDİRME (RESULTS OF APPLICATION SOFTWARE AND DISCUSSION)}

IEEE 802.15.4 haberleşmesi karşıllklı olarak veri iletiminde bulunan noktadan noktaya (peer to peer) düğümler için test edilmiştir. Haberleşmede gürültüsüz ortamdan (çip hata oranı=0), $\% 0.25$ değerine kadar çip hata oranını oluşturan 26 farklı gürültülü ortamda maksimum uzunluklu IEEE 802.15.4 çerçeveleri iletilmiştir. Uygulamada her bir gürültü oranında $4 / 5,4 / 6,4 / 7, \ldots, 4 / 58$ yayılım faktörleri için 30 defa koşturulmuş ve bunlara ilişkin ortalama değerler alınmıştır. Her bir gönderimde 102 oktet mesaj bilgisini içeren 128 oktet çerçeve uzunluğu kullanılmıştır. Bu çerçeveler PAN adresleri içermeyecek şekilde kısa kaynak ve hedef adreslemesini (short source and destination adressing mode) destekler. IEEE 802.15.4 slotsuz CSMA için yapılan konfigürasyonda yer alan bazı hesaplar ve parametreler Şekil 6'da verilmiștir.

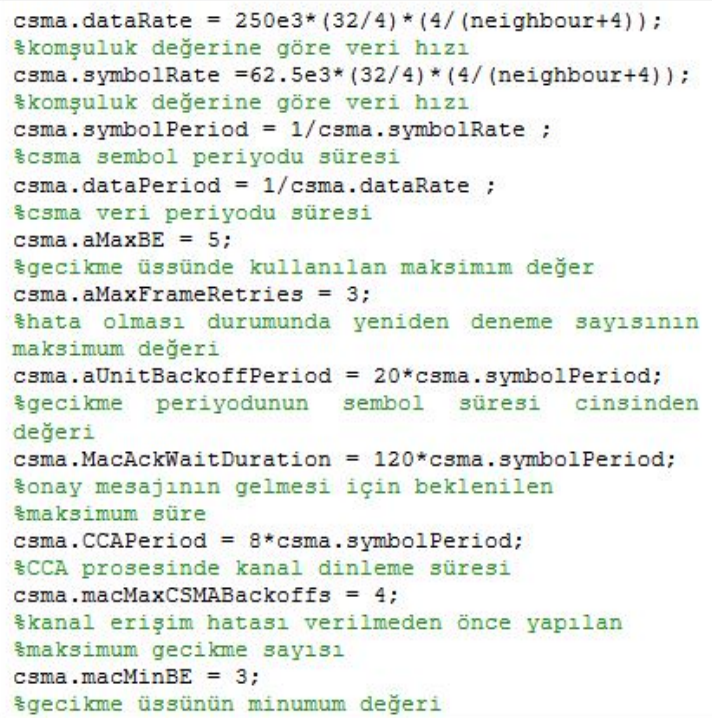

Şekil 6. CSMA konfigürasyonu (CSMA configuration)

Her bir çip hata oranı için elde edilen maksimum verimliliğe göre en iyi veri aktarım oranına sahip olan ortalama yayılım faktörü değeri Tablo 3' de verilmiştir. IEEE 802.15 .4 kodlaması $1 / 8$ oranına sahiptir. 4/5, 4/6, 4/7, .. , 4/58 kodlamalarının standart DSSS kodlamasina kiyasla teoride (4/58) / $(4 / 32) \cong 0,552$ kat daha yavaştır ve $(4 / 5) /(4 / 32) \cong$ 6,4 kat daha hizlıdır. Ancak pratikte bu hesaba gürültünün bozduğu iletimlerde dâhil edilmelidir. Şekil 7'de veri aktarım oranı çip hata oranı grafiği verilmiştir. Grafiğe göre çip hata oranı arttıkça verimlilik miktarı düşmüştür. Çip hata oranının 0 olduğu ortamda 857621,44 değerinde aktarım elde edilirken hata oranı 0.25 ' e yükseltildiğinde bu değer 602,09 ' a düşmüştür. $\mathrm{Bu}$ nedenle düşüş oranı $\frac{857621,44}{602,09} \cong 1424,40$ kat düşmüştür. Her bir hata oranı için adaptif yayılım faktörlerinin ve IEEE 802.15.4 standardı 4/32 yayılım faktörünün kıyaslanması Şekil 7' de verilmiştir. Şekil 7' de görüldüğü üzere üretilen adaptif gürültü dizileri her bir çip hata oranı için daha yüksek veri aktarım oranına sahiptir. Özellikle çip hata oranı 0 ile 0.05 değerleri arasında veri aktarım oranı DSSS(4/32) kodlamasına göre çok yüksektir. Çip hata oranı 0 için $\frac{857621,4405}{145662,3} \cong 6$ kat daha yüksek veri aktarım oranı elde edilmiştir.

Tablo 3. Her bir çip hata oranı için ortalama yayılım faktörü (Average spreading factor for each chip error rate)

\begin{tabular}{|c|c|c|}
\hline Sıra & Çip Hata Oranı & Yayılım Faktörü \\
\hline 1 & 0 & 5 \\
\hline 2 & 0,01 & 12 \\
\hline 3 & 0,02 & 16 \\
\hline 4 & 0,03 & 16 \\
\hline 5 & 0,04 & 20 \\
\hline 6 & 0,05 & 21 \\
\hline 7 & 0,06 & 24 \\
\hline 8 & 0,07 & 26 \\
\hline 9 & 0,08 & 28 \\
\hline 10 & 0,09 & 30 \\
\hline 11 & 0,1 & 32 \\
\hline 12 & 0,11 & 36 \\
\hline 13 & 0,12 & 39 \\
\hline 14 & 0,13 & 43 \\
\hline 15 & 0,14 & 47 \\
\hline 16 & 0,15 & 50 \\
\hline 17 & 0,16 & 54 \\
\hline 18 & 0,17 & 56 \\
\hline 19 & 0,18 & 57 \\
\hline 20 & 0,19 & 57 \\
\hline 21 & 0,2 & 57 \\
\hline 22 & 0,21 & 58 \\
\hline 23 & 0,22 & 58 \\
\hline 24 & 0,23 & 58 \\
\hline 25 & 0,24 & 56 \\
\hline 26 & 0,25 & \\
\hline & & 56 \\
\hline
\end{tabular}

Hata oranına göre elde edilen maksimum verimlilik (veri aktarım oranı) değerleri Tablo 4' de verilmiştir. Tabloda ilk sütun hata oranlarını, ikinci ve üçüncü sütunlar ise sirasiyla önerilen metodun(4/..) ve klasik(4/32) çip dizisinin ürettiği veri aktarım oranını vermektedir. Uygulama 0 ile 0.25 aralığındaki çip hata oranları için 30 koşturma sonucunda maksimum verimliliği sağlayan adaptif sözde rasgele gürültü dizisinin uzunluğunu 39 (yaklaşık değer) olarak belirlemiştir. $\mathrm{Bu}$ alt bölümde paket gönderimi için adaptif sözde rasgele dizileri 39 bit uzunluğunda oluşturulmuş ve her bir çip hata oranı için 128 paket gönderilmiştir. Çip hata oranları $0,0.3,0.6$ ve 0.9 değerleri için 4/32 yayılım faktörüne sahip sözde rasgele gürültü dizileri, 4/39 yayılım faktörüne sahip gürültü dizilerine göre daha hızlı veri iletimini sağlamışlardır. 
Tablo 4. Çip hata oranına yönelik maksimum verimlilik tablosu (Maximum efficiency table for chip error rate)

\begin{tabular}{|c|c|c|}
\hline $\begin{array}{c}\text { Çip Hata } \\
\text { Oranı }\end{array}$ & $\begin{array}{c}\text { Maksimum } \\
\text { Veri aktarım } \\
\text { oranı (4/..) } \\
\end{array}$ & $\begin{array}{c}\text { Maksimum } \\
\text { Veri aktarım } \\
\text { oranı }(4 / 32) \\
\end{array}$ \\
\hline 0 & 857621.44 & 145662.30 \\
\hline 0.01 & 362824.14 & 146118.72 \\
\hline 0.02 & 280207.75 & 144822.13 \\
\hline 0.03 & 257575.75 & 144373.71 \\
\hline 0.04 & 221125.10 & 142732.22 \\
\hline 0.05 & 199637.61 & 145015.14 \\
\hline 0.06 & 182334.91 & 145357.41 \\
\hline 0.07 & 171699.67 & 136136.12 \\
\hline 0.08 & 156724.35 & 136107.82 \\
\hline 0.09 & 142279.96 & 138498.73 \\
\hline 0.1 & 131398.98 & 118686.61 \\
\hline 0.11 & 120159.03 & 100977.60 \\
\hline 0.12 & 111010.73 & 82889.27 \\
\hline 0.13 & 102080.55 & 55241.98 \\
\hline 0.14 & 94048.49 & 33543.29 \\
\hline 0.15 & 87828.11 & 16599.87 \\
\hline 0.16 & 81231.92 & 5724.81 \\
\hline 0.17 & 78018.93 & 1903.13 \\
\hline 0.18 & 69678.17 & 536.62 \\
\hline 0.19 & 57622.92 & 0 \\
\hline 0.2 & 42674.79 & 0 \\
\hline 0.21 & 31613.79 & 0 \\
\hline 0.22 & 16260.96 & 0 \\
\hline 0.23 & 7206.93 & 0 \\
\hline 0.24 & 2503.99 & 0 \\
\hline 0,25 & 602,0917153 & 0 \\
\hline
\end{tabular}

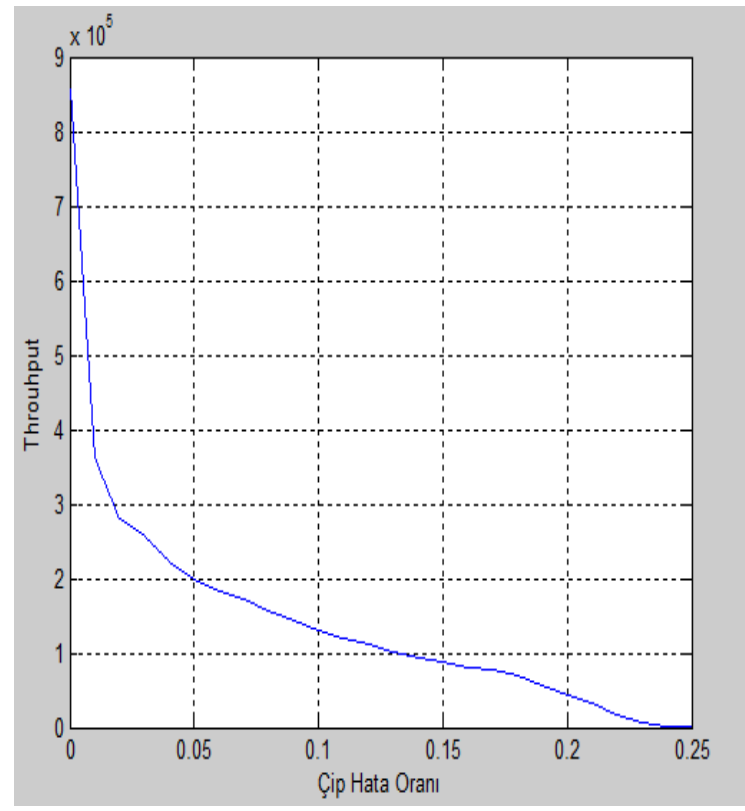

Şekil 7. Çip hata oranı - veri aktarım oranı grafiği (Chip error rate - throughput graph)

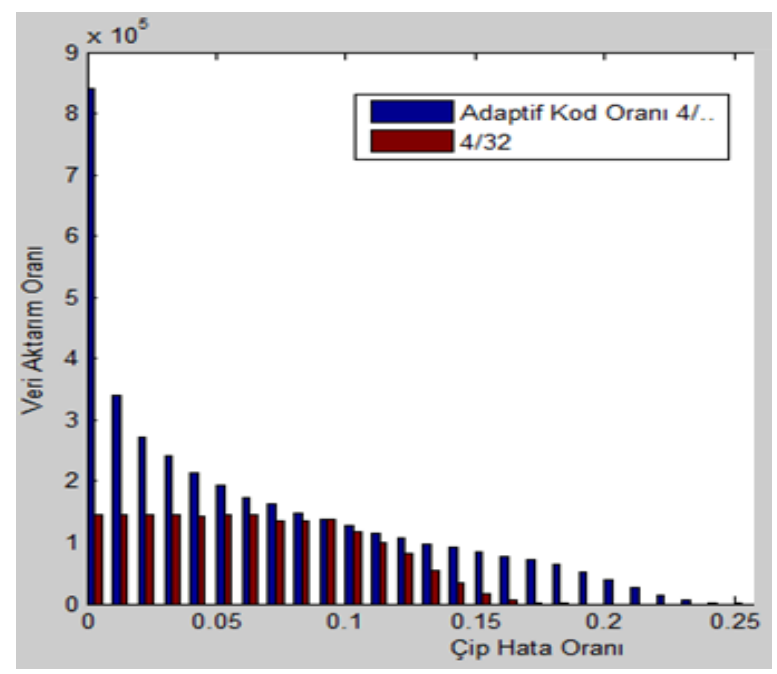

Şekil 8. Her bir çip hata oranı için adaptif yayılım faktörleri ve DSSS(4/32) karşılaştırması (Adaptive spreading factors for each chip error rate and comparison for $\operatorname{DSSS}(4 / 32))$

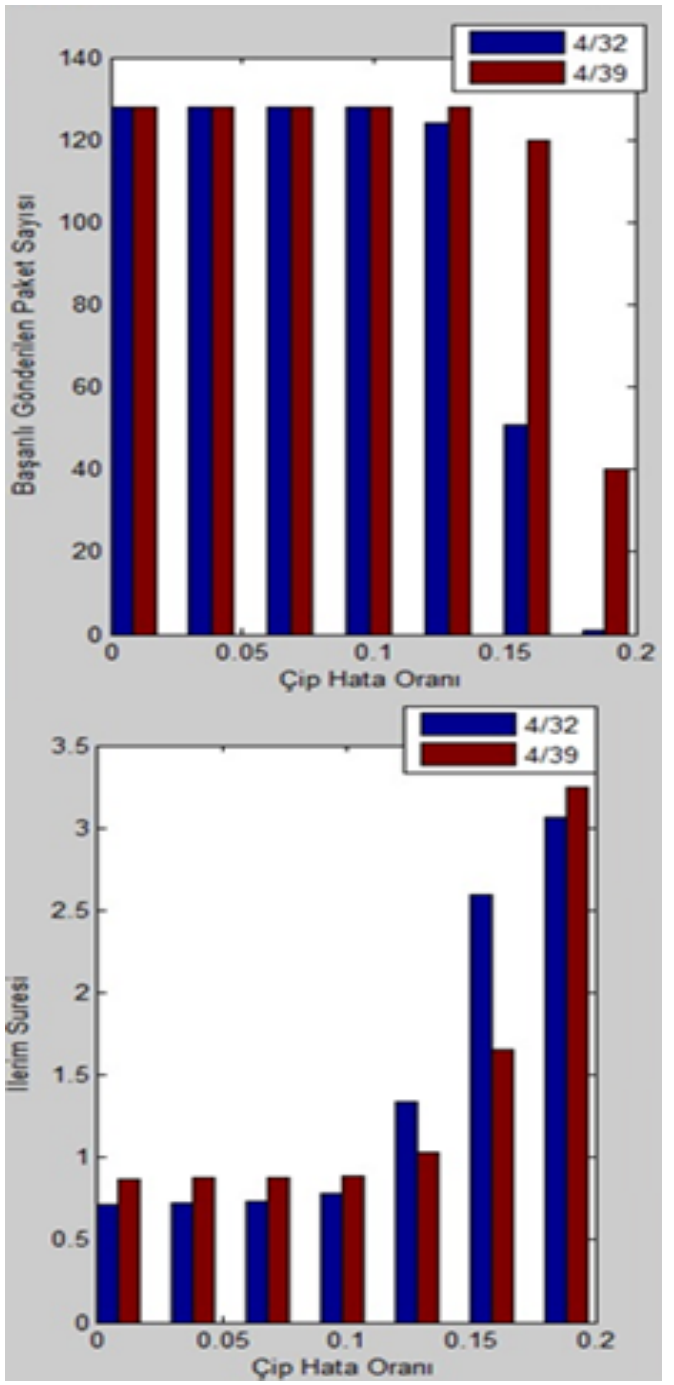

Şekil 9. 4/32 ve 4/39 yayllım faktörlerine sahip gürültü dizileri karşılaştırması (comparison for noise sequences with spreading factors of $4 / 32$ and $4 / 39$ ) 
Adaptif yayılım faktörüne sahip gürültü dizileri 4/32 yayılım faktörüne sahip gürültü dizilerine göre daha çok paket göndermeyi başarmıştır. $\mathrm{Bu}$ sonuçlara bakarak ortamdaki gürültü seviyesi arttırıldığında bit uzunluğu arttırılarak üretilen sözde rasgele gürültü dizilerinin paket gönderiminde daha başarılı olduğu söylenebilir. Şekil 8 ve Şekil 9 'da 4/32 ve 4/39 gürültü dizilerinin karşılaştırılması verilmiştir. Şekil 8' de gürültü oranının veri aktarım oranına (throughput) etkisinin negatif olduğu açıktır. Adaptif yaklaşımın ise bu negatif etkiden çok daha az etkilendiği Şekil 8 ve Şekil 9' da görülmektedir.

\section{SONUÇLAR (CONCLUSIONS)}

IEEE 802.15.4 protokolü, basit karmaşıklıktaki donanım ve yazılımlarla gerçekleşebilen, düşük veri iletimi sağlayan haberleşme standardıdır. Gürültülü ortamlarda veri iletimi daha başarılıdır. Ancak standartta kullanılan DSSS(32,4) modülasyon tekniği, ortamdaki gürültü seviyesinin değişmesinden bağımsız olarak paket gönderir. Dolayısıyla gürültü seviyesi azken daha yüksek bir yayılım faktörü kullanır. Adaptif gürültü dizilerinin sahip olduğu bir yapıda paket gönderimi nispeten daha başarılı olur. Performansta artışın hangi durumlarda ve ne oranlarda olduğu uygulama çalışmalarıyla elde edilmiştir.

Ortamdaki gürültü seviyeleri gözetilerek üretilen adaptif PN dizileriyle IEEE 802.15.4 protokolünde MAC parametrelerinde dikkate değer artışlar gözlenmiştir. Ortamdaki gürültü seviyesi az olduğu durumlarda yaklaşık 6 kata kadar başarı sağlanmıştır.

Gelecek çalışmalarda, adaptif yayılım faktörünü üretme mekanizmasının gerçek-zamanlı sistemlere uygun yapıda modellenmesi hedeflenmektedir.

\section{KAYNAKLAR (REFERENCES)}

1. Öztaş, Ş., Belen, M., Kaya, İ. ve Kaya, A., "Zigbee Teknolojisi Kullanılarak Kablosuz Kafe Otomasyon Sistemi Tasarımı", ElektrikElektronik Bilgisayar Sempozyumu (FEEB 2011), Elazığ/Türkiye, 203-206, 5-7 Ekim 2011.

2. $\mathrm{Ci}, \mathrm{S}$. ve Sharif, H., "A Link Adaptation Scheme For Improving Throughput in the IEEE 802.11 Wireless LAN", in Proceedings of the 27th Annual IEEE Conference on Local Computer Networks, Tampa, USA, 205-210, 6-8 November 2002.

3. Lin, Y., "Efficient Dynamic Frame Aggregation in IEEE 802.11s Mesh Networks", International Journal of Communication Systems, Cilt 10, 1319-1338, 2009.

4. Xia, Q. ve Hamdi, M., "Contention Window Adjustment for IEEE 802.11 WLANs: a Controltheoretic Approach", in Proceedings of IEEE on
Communications (ICC06), Istanbul, Turkey, 3923-3928, 11-15 June 2006.

5. Nafaa, A., "Sliding Contention Window (SCW): Towards Backoff Range-based Service Differentiation Over IEEE 802.11 Wireless LAN Networks", IEEE Network, Cilt 19, No 4, 45-51, 2005.

6. Kim, B.S., Fang, Y. ve Wong., T.F., "Throughput Enhancement Through Dynamic Fragmentation in Wireless LANs", IEEE Transactions on Vehicular Technology, Cilt 54, No 4, 14151425, 2005.

7. $\mathrm{Ci}, \mathrm{S}$. ve Sharif, H., "Adaptive Approaches to Enhance Throughput of IEEE 802.11 Wireless LAN With Bursty Channel", in Proceedings of the 25th Annual IEEE Conference on Local Computer Networks, Tampa, USA, 44-45, 8-10 November 2000.

8. Qiao, D. ve Choi, S., "Goodput Enhancement of IEEE 802.11a Wireless LAN Via Link Adaptation", in Proceedings of IEEE International Conference on Communications, Helsinki, Finland, 1995-2000, 11-14 June 2001.

9. Qiao, D., Choi, S. ve Shin, K.G., "Goodput Analysis and Link Adaptation for IEEE 802.11a Wireless LANs", IEEE Transactions on Mobile Computing, Cilt 1, No 4, 278-291. 2002.

10. Foh, C. ve Zukerman, M., "Performance Analysis of the IEEE 802.11 MAC Protocol", in Proceedings of EW 2002 Conference, Florence, Italy, 184-190, 25-28 February 2002.

11. Li, F., "Tools and Techniques for Measurement of IEEE 802.11 Wireless Networks", in Proceedings of the 4th International Symposium on Modeling and Optimization in Mobile, Ad Hoc and Wireless Networks, Boston, USA, 1-8, 3-6 April 2006.

12. Sato, T. , Kawabe, M., Kato, T. ve Fukasawa, A., "Throughput Analysis Method for Hybrid ARQ Schemes Over Burst Error Channels", IEEE Transactions on Vehicular Technology, Cilt 42, 110-118, 1993.

13. Wang, H.S. ve Chang, P.C., "On Verifying the First-order Markovian Assumption for a Rayleigh Fading Channel Model", IEEE Transactions on Vehicular Technology, Cilt 45, 353-357, 1996.

14. Shu, F. ve Sakurai, T., "A New Analytical Model For the IEEE 802.15.4 CSMA-CA Protocol", Computer Networks, Cilt 55, 2576-2591, 2011.

15. Goyal, M., "A Stochastic Model For Beaconless IEEE 802.15.4 MAC Operation", Computer Communications, Cilt 34, 1460-1474, 2011.

16. Misic, J., Shafi, S. ve Misic, V., "The Impact of MAC Parameters on the Performance of 802.15.4 PAN", Ad Hoc Networks, Cilt 3, 509-528, 2005.

17. Zhu, J., Tao, Z. ve Lv, C., "Performance Evaluation for a Beacon Enabled IEEE 802.15.4 Scheme With Heterogeneous Unsaturated Conditions", International Journal of 
Electronics and Communications (AEÜ), Cilt 66, 93-106, 2012.

18. Fukawa, K., "Packet-Error-Rate Analysis Using Markov Models of the Signal-To-Interference Ratio for Mobile Packet Systems", IEEE Transactions on Vehicular Technology, Cilt 61, No 6, 2517-2530, 2012.

19. Badia, L., Baldo, N., Levorato, M. ve Zorzi, M., "A Markov Framework For Error Control Techniques Based on Selective Retransmission in Video Transmission Over Wireless Channels", IEEE Journal on Selected Areas in Communications (JSAC), Cilt 28, No 3, 488500, 2010.

20. Lee, C.Y., Cho, H., Hwang, G.U., Doh, Y. ve Park, N., "Performance Modeling and Analysis of IEEE 802.15.4 Slotted CSMA/CA Protocol With ACK Mode", International Journal of Electronics and Communications, Cilt 65, No 2, 123-131, 2011.

21. Park, P., "Duty-cycle Optimization For IEEE 802.15. 4 Wireless Sensor Networks", Computer Communications, Cilt 10, No 1, 12-44, 2013.

22. Marco, P.D., Park, P. ve Johansson, K. H., "Analytical Modeling of Multi-hop IEEE 802.15.4 Networks", IEEE Transactions on Vehicular Technology, Cilt 61, No 7, 31913208, 2012.
23. Wang, F., Li., D. ve Zhao, Y., "Analysis of CSMA/CA in IEEE 802.15.4", IET Communications, Cilt 5, 2187-2195, 2011.

24. Sönmez, İ., IEEE 802.15.4 Standardına Uyumlu RF Haberleşme Uygulaması, Yüksek Lisans Tezi, Yıldız Teknik Üniversitesi, Fen Bilimleri Enstitüsü, 2007.

25. IEEE P80215 Working Group, "IEEE 802.15.4 2003: Wireless Medium Access Control (MAC) and Physical Layer (PHY) Specifications for Low Rate Wireless Personal Area Networks (LRWPANs)", IEEE Protocol Specifications, (erişim: 14 Ağustos 2015), http://standards. ieee.org/getieee802/download/802.15.4-2003. pdf, 2003.

26. Goyal, M., Prakash, S., Xie, W., Bashir, Y., Hosseini, H., Durresi, A. ve Wisconsin, U., "Evaluating the Impact of Signal To Noise Ratio on IEEE 802.15.4 PHY-Level Packet Loss Rate", in Proceedings of the 13th International Conference on Network-Based Information Systems, Takayama, Japan, 279-284, 14-16 September, 2010.

27. Mathworks Support Team, "Bit Error Rate (BER)", Mathworks Support Web Site, (erişim: 4 Şubat 2014), www.mathworks.com/ help/comm/ug/bit-error-rate-er.html\#bq422mf, 2014. 